\title{
Left atrial appendage closure for "primary primary" prevention during percutaneous closure of septal defects in patients with large atria but no atrial fibrillation
}

\author{
Shingo Kuwata ${ }^{1}$, Juliane Vierecke ${ }^{2}$, Steffen Gloekler ${ }^{3}$, \\ Francesco Maisano ${ }^{1}$, Bernhard Meier $^{2}$, Fabian Nietlispach ${ }^{1}$ \\ ${ }^{1}$ University Heart Center, Zurich, Switzerland \\ ${ }^{2}$ University Hospital Bern, Bern, Switzerland \\ ${ }^{3}$ Cardiology, Schwarzwald-Baar Klinikum, Villingen-Schwenningen, Germany
}

\begin{abstract}
Background: Percutaneous atrial septal defect (ASD) closure is a routine procedure to prevent right ventricular failure, pulmonary hypertension, or paradoxical embolism. The latter is the typical reason for percutaneous patent foramen ovale $(P F O)$ closure. Atrial enlargement represents a risk for developing atrial fibrillation $(A F)$. Percutaneous left atrial appendage (LAA) closure is emerging as a preventive therapy for patients in $A F$ who suffered from a previous stroke or bleeding (secondary prevention) or patients without previous stroke or bleeding (primary prevention). Percutaneous septal closure, particularly that of large ASDs, may inhibit future percutaneous left atrial access when required for $L A A$ closure. Reported herein is the feasibility and safety of concomitant percutaneous closure of the $L A A$ and a septal shunt, mostly large ASDs, in patients without AF, in the sense of "primary primary" preventive LAA closure. The first "primary" relates to "in anticipation of $A F$ " and potentially also for "for prevention of $A F$ ". The second "primary" relates to "prevention of stroke or bleeding".

Methods: Thirteen consecutive patients, older than 40 years without any clinical or electrocardiographic evidence of $A F$, underwent percutaneous closure of large ASDs or PFOs in the presence of enlarged atria at the university hospitals of Bern and Zurich between April 2013 and June 2015. They concomitantly received "primary primary" preventive LAA closure after informed consent.

Results Mean patient age was $58 \pm 9$ years ( $46 \%$ male). Procedural success was achieved in all patients and no major adverse events occurred acutely or during the following $2.0 \pm 0.8$ years. No patient developed $A F$.

Conclusions: Concomitant closure of ASD or PFO in the presence of enlarged atria and LAA for "primary primary" prevention appears feasible and safe but has yet to prove its justification. (Cardiol J 2018; 25, 2: 179-187)
\end{abstract}

Key words: atrial fibrillation, atrial septal defect closure, patent foramen ovale closure, left atrial appendage closure

Address for correspondence: Fabian Nietlispach, MD, PhD, University Heart Center, University Hospital of Zurich, Rämistrasse 100, CH-8091 Zürich, Switzerland, tel: +41 4425532 98, fax: +41 4425544 46, e-mail: Fabian.Nietlispach@usz.ch

Received: 26.06.2017 Accepted: 22.08.2017 


\section{Introduction}

Atrial septal defect (ASD) is one of the most frequent congenital heart defects [1]. While the only risk of small ASDs is paradoxical embolism, sequelae of larger ASDs comprise pulmonary hypertension, congestive heart failure, and atrial arrhythmia, in particular atrial fibrillation (AF) [2-5]. Percutaneous ASD closure is the treatment of choice for ASDs to prevent such adverse outcome and to improve exertional dyspnea [6-8]. Percutaneous ASD closure is an overall safe procedure but it carries a potential risk for developing AF by device irritation of the septum [9-11]. Although such new onset AF typically occurs only in the first few weeks after device implantation [9, 12], patients undergoing percutaneous closure of large ASDs are temporarily at a double risk for developing $\mathrm{AF}, 1$ ) due to septal device irritation and 2) due to the preexisting atrial enlargement. Subsequent reduction of atrial diameters after shunt elimination reduces the risk for AF. A net benefit in that respect can be expected but the overall risk for $\mathrm{AF}$ of such patients remains elevated. The situation is similar for patients with a patent foramen ovale (PFO) and enlarged atria.

In $\mathrm{AF}$, left atrial appendage (LAA) closure has emerged as an alternative to medical therapy in the form of chronic oral anticoagulation (COA) for stroke or bleeding prevention. While closure of the LAA comes at the price of 2-7\% procedural adverse events, it shows a significant reduction in major bleeding events at a similar stroke rate compared to COA [13-16]. After 4 years of follow-up, closure of the LAA even showed significantly improved survival in randomized comparisons $[17,18]$.

Closure of the LAA so far has been exclusively performed in patients with documented $\mathrm{AF}$, either for primary prevention (patients with $\mathrm{AF}$ without previous embolic ischemic event or bleeding) or secondary prevention (patients with AF with a previous embolic ischemic event or bleeding).

Percutaneous closure of septal defects, particularly large ASDs, hinders future percutaneous transseptal left atrial access although it remains possible at least in the case of smaller ASD [19] or PFO devices [20]. Even if such patients never had $\mathrm{AF}$, their risk for developing AF during follow-up is 2-fold higher after ASD closure than that of the general population [21] and also increased after PFO closure in the presence of enlarged atria.

Herein reported is the first experience with concomitant percutaneous closure of LAA at the time of closure of ASDs or PFOs in the presence of enlarged atria in patients without history of AF. They were considered at increased risk for later AF, particularly in the initial phase after septal shunt closure due to septal device irritation while atrial dimensions have not yet shrunk after volume unload. It is referred to in this procedure as "primary primary" preventive LAA closure, i.e., LAA closure for primary prevention (in anticipation of $\mathrm{AF}$ or even preventing $\mathrm{AF}$ ) and for primary prevention of stroke or bleeding, should AF occur.

\section{Methods}

\section{Population}

This is a retrospective study of consecutive patients ( $\geq 40$ years) undergoing percutaneous closure of a large ASD $(\geq 20 \mathrm{~mm})$ or a PFO with large atria (left atrium $[\mathrm{LA}] \geq 40 \mathrm{~mm}$, parasternal long axis) and at the same time of the LAA. Patients neither had clinical or electrocardiographic signs of prior AF nor an AF associated embolic ischemic or bleeding event. In the patients with prior ischemic events, AF had been excluded by long-term electrocardiogram (ECG). Patients consented to both procedures (ASD closure and LAA closure) separately and also to data collection. The potential benefits and risks of "primary primary" preventive LAA closure was discussed extensively with the patients. The local institutional review boards approved data collection of clinically indicated investigations during follow-up. The procedures were performed at the university hospitals of Bern and Zurich. Demographic and clinical characteristics, including risk for stroke and major bleeding $\left(\mathrm{CHA}_{2} \mathrm{DS}_{2}\right.$-VASc score, HAS-BLED score), procedural data, and outcome data of in-hospital and follow-up periods were captured from medical records and patient contact.

\section{Procedure}

All procedures were performed under fluoroscopy guidance without echocardiography [15, 22, 23]. All patients had had transesophageal echocardiography (TEE) during their workups. Computer tomography was not used. Oral antibiotics (2-3 doses) were given before and after the procedure and all patients received 5000 units of intravenous heparin at the beginning of the procedure. All procedures were done using Amplatzer devices (Amplatzer Cardiac Plug (ACP), Amplatzer Amulet Occluder, Amplatzer Septal Occluder (ASO), Amplatzer Cribriform Occluder, or Amplatzer PFO Occluder, Abbott, St. Paul, MN, USA). Figures 1-4 provide examples. LAA closure 


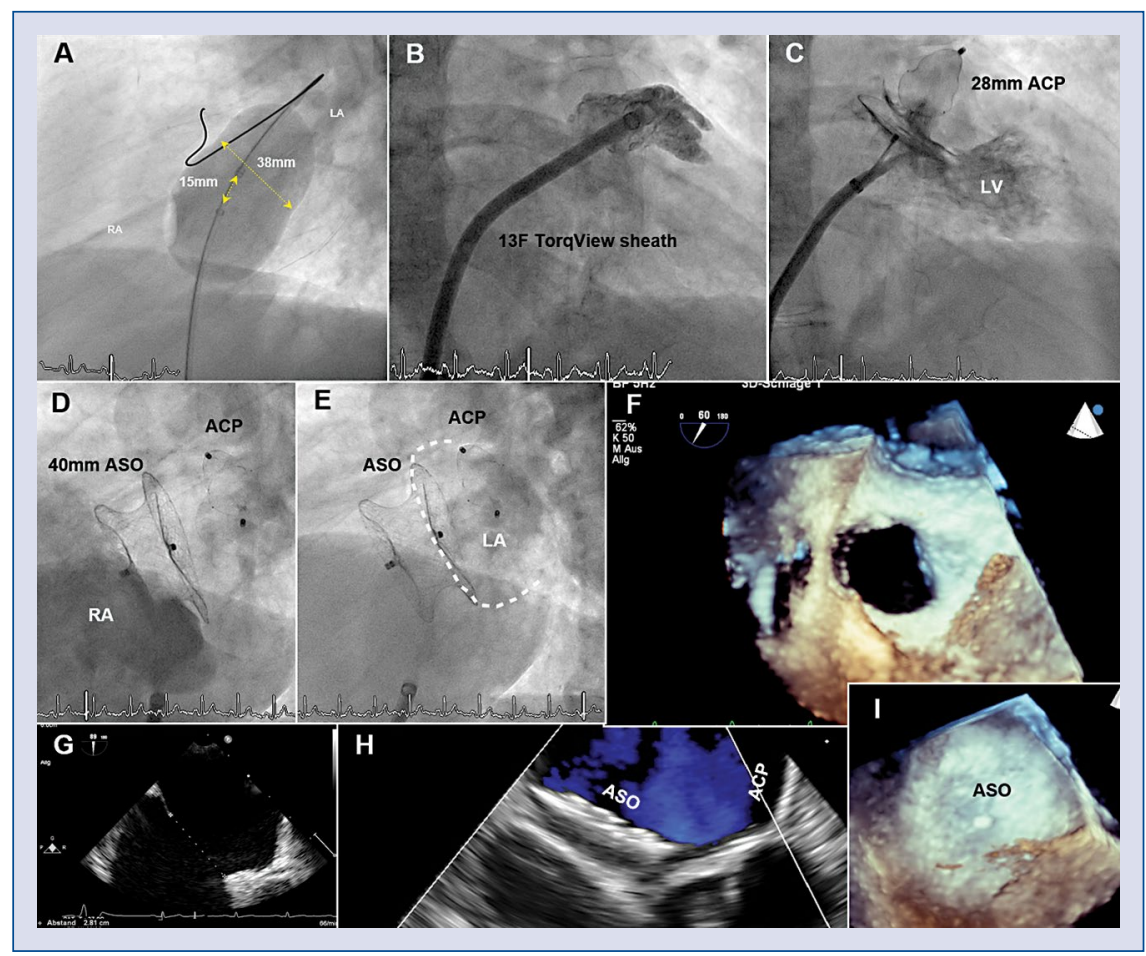

Figure 1. Simultaneous closure of the left atrial appendage (LAA) and an atrial septal defect (ASD) in a 55-year-old male with exertional dyspnea and a newly detected large ASD secundum but no history of atrial fibrillation. A. Overinflated $34 \mathrm{~mm}$ Amplatzer sizing balloon in ASD, measuring $38 \mathrm{~mm}$ at the waist representing the ASD; B. Contrast medium injection into the LAA through a 13 French (F) TorqView sheath in a right anterior oblique projection; C. Implantation of a $28 \mathrm{~mm}$ Amplatzer Cardiac Plug (ACP) through this sheath; D. Implantation of a $40 \mathrm{~mm}$ Amplatzer Septal Occluder (ASO) through the same sheath in a left anterior oblique projection. The right atrium (RA) is filled with contrast medium; E. Same view during the levophase with the left atrium (LA) delineated (dotted line) by contrast medium; F. Three-dimensional (3D) transesophageal echocardiography (TEE) before the procedure depicting the ASD; G. Two-dimensional (2D) TEE before the procedure indicating an ASD diameter of $28 \mathrm{~mm} ; \mathbf{H}$. 2D TEE at 11-month follow-up depicting the ASD and LAA occluders; I. 3D TEE at 11-month follow-up. The ASO occludes the ASD completely without evidence of thrombus.

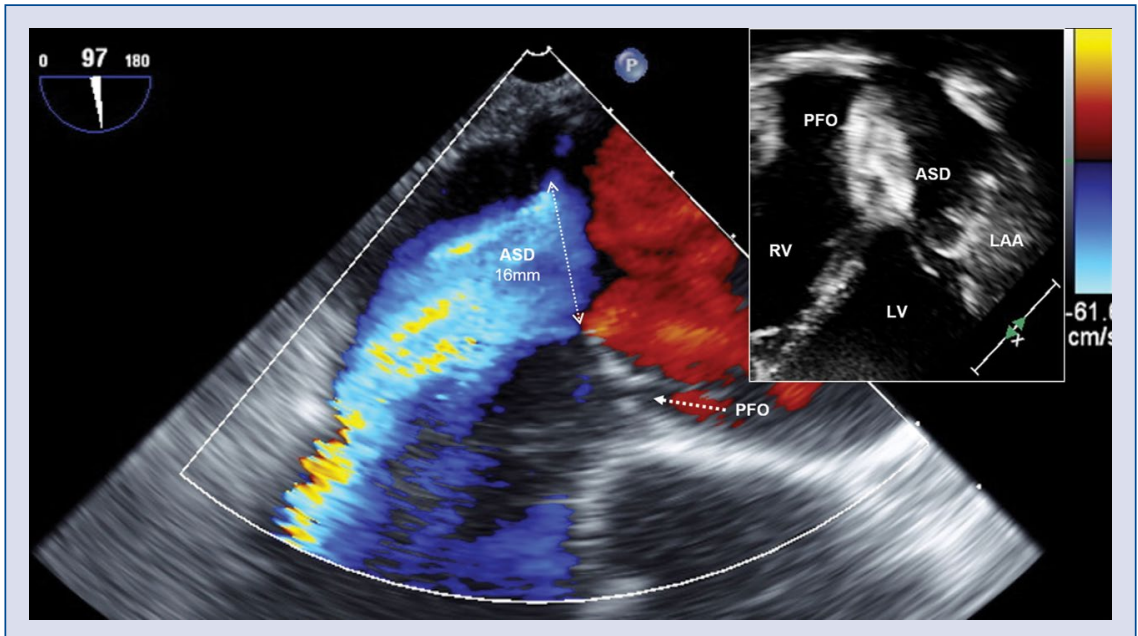

Figure 2. Transesophageal echocardiography of a 53-year-old woman with exertional dyspnea and chest pain and a newly detected secundum atrial septal defect (ASD) as well as a patent foramen ovale (PFO) but no history of atrial fibrillation. The coronary arteries were normal on the incidental angiogram. The insert shows the result after simulatenous device closure of ASD (26 mm Amplatzer Septal Occluder), PFO (30 mm Amplatzer Cribriform Occluder), and left atrial appendage (LAA, $22 \mathrm{~mm}$ Amplatzer Amulet) Occluder; LV — left ventricle; RV — right ventricle. 


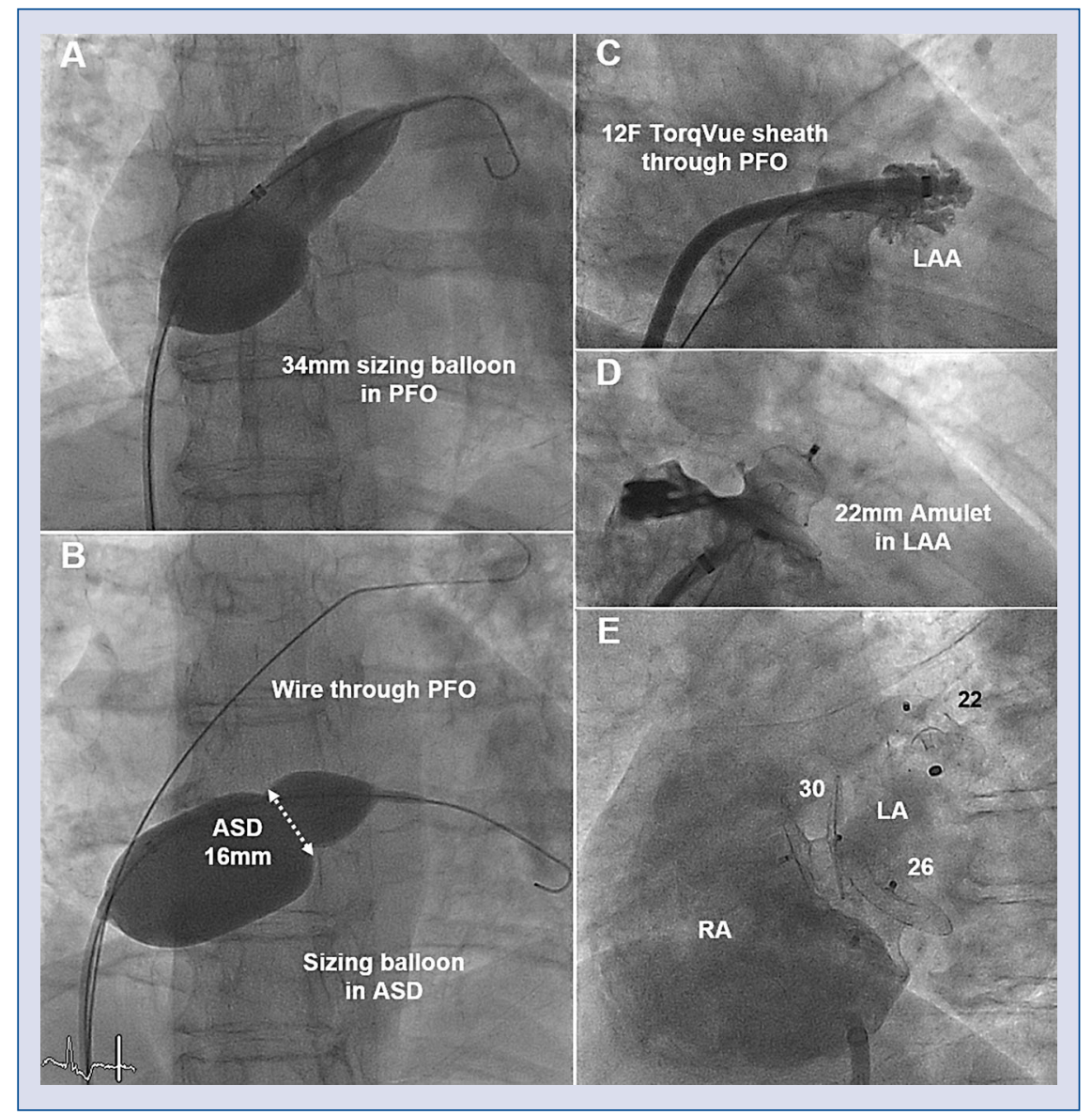

Figure 3. Simultaneous closure of patent foramen ovale (PFO), atrial septal defect (ASD), and left atrial appendage (LAA) in patient of Figure 2; A. Sizing balloon in PFO; B. Sizing balloon in ASD, wire through PFO; C. Contrast medium injection into LAA through 12 French (F) TorqVue sheath in a right anterior oblique projection; D. LAA occluded (with $22 \mathrm{~mm}$ Amplatzer Amulet Occluder); E. Final result: LAA occluded with $22 \mathrm{~mm}$ Amplatzer Amulet Occluder (22), then PFO occluded with $30 \mathrm{~mm}$ Amplatzer Cribriform Occluder (30), and finally ASD occluded with $26 \mathrm{~mm}$ Amplatzer Septal Occluder (26). Left anterior oblique projection; LA — left atrium; RA — right atrium.

was performed before septal closure. After crossing the septal defect from the right femoral vein, a 0.035” Backup wire (Boston Scientific, Natick, MA, USA) was placed in the LA. A $34 \mathrm{~mm}$ Amplatzer sizing balloon was placed across ASDs and inflated to aid eyeball sizing of the defect. The balloon was removed. Over the Backup wire, the 14 French (F) $45^{\circ} \times 45^{\circ}$ Amplatzer TorqVue delivery sheath was introduced. The LAA was engaged in a right anterior oblique (RAO) view and contrast injections were performed in RAO caudal and cranial views. Sizing was done by eyeballing the outer diameter of the delivery sheath $(12 \mathrm{~F}: 4.5 \mathrm{~mm}, 13 \mathrm{~F}: 5.0 \mathrm{~mm}$, and $14 \mathrm{~F}: 5.5 \mathrm{~mm}$ ) served as reference. About $20-50 \%$ oversizing was the rule [15]. Stability of the device was assessed by a tug test and by angiographic imaging with contrast medium injections in 2 views showing the device in profile without overlap of the 2 device components. After release of the LAA occluder, the TorqVue sheath was left in the LA and the appropriately sized septal occluder (aiming for at least 30\% oversizing in ASDs) was attached to the same delivery cable and introduced through the sheath. In case of ASDs, the left atrial disc was fully and the right atrial disc partially deployed in the LA. This technique forces the ASO to self-center in the defect. The pulling of the right disc trough, the defect was observed based on the migrating of the lower device indentation in a left anterior oblique (LAO) projection before the right atrial disc was fully deployed. Correct device position was confirmed by LAO contrast medium injections and a robust wiggle of the device. The sheath was removed and the puncture site was manually compressed by the patient unless a simultaneous arterial puncture mandated compression 


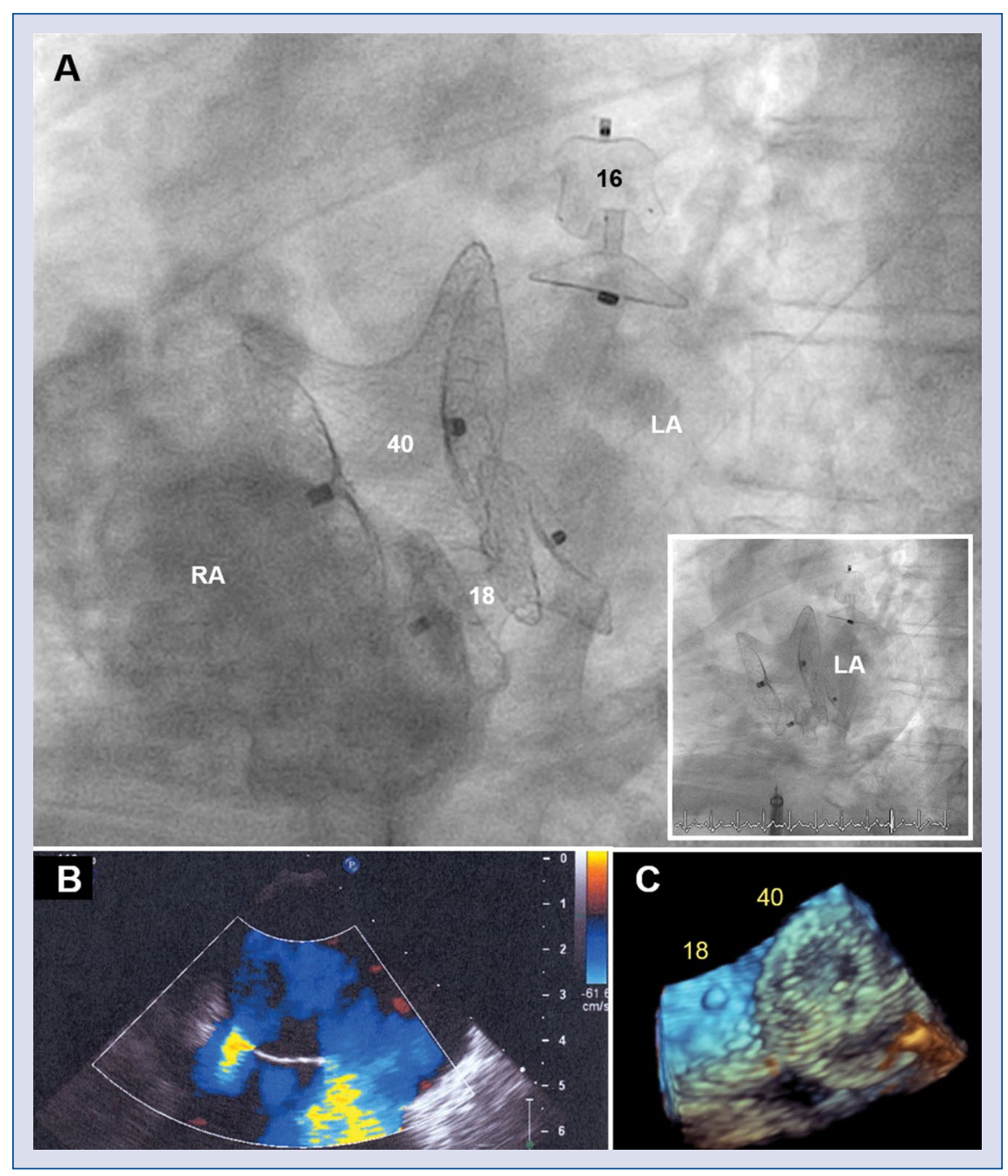

Figure 4. Simultaneous closure of the left atrial appendage (LAA) and a newly detected double atrial septal defect (ASD) in a 64-year-old male with dyspnea but no atrial fibrillation. All devices were implanted through the same 14 French TorqVue sheath; A. $40 \mathrm{~mm}$ Amplatzer Septal Occluder (ASO, 40), $18 \mathrm{~mm}$ ASO (18), and $16 \mathrm{~mm}$ Amplatzer Amulet Occluder (16) depicted in a in a left anterior oblique projection. The right atrium (RA) and in the insert the left atrium (LA) are filled with contrast medium; B. Double ASD in a transesophageal echocardiography (TEE) before the intervention; C. Double ASD in a three-dimensional TEE 4 months after the intervention with the two ASOs in place.

by a medical person. Before discharge the same or the following day, transthoracic echocardiography was performed to assure the correct position of the devices and to exclude pericardial effusion. There were no recommendations for physical restrictions of any sort.

Post interventional antithrombotic regimen comprised clopidogrel $75 \mathrm{mg}$ for 1-3 months and acetylsalicylic acid $100 \mathrm{mg}$ for $5-6$ months. Control TEE was recommended after 3-6 months to assess for device thrombi and residual shunts or leaks.

\section{Outcome}

The composite endpoint was defined according to Valve Academic Research Consortium (VARC)-2 criteria [24]. The primary safety endpoint was defined as death related to closure of the LAA, procedure-related ischemic stroke, hemorrhagic stroke, cardiac tamponade, need for urgent surgery (e.g., due to embolization of the device, repair of procedure-related injury, or bleeding) and major or life-threatening bleeding (according to VARC-2 criteria). The primary efficacy endpoint was defined as stroke (non-disabling, disabling, ischemic, or hemorrhagic), systemic embolization, or cardiovascular or unexplained death. New-onset of AF was looked for clinically and by (24-h) ECG. Clinical follow-up data were obtained reviewing medical records and by contacting patients. 
Table 1. Baseline characteristics ( $n=13$ ).

\begin{tabular}{|c|c|}
\hline \multicolumn{2}{|l|}{ Characteristics } \\
\hline Age [years] & $58.2 \pm 9.1$ \\
\hline Body mass index $\left[\mathrm{kg} / \mathrm{m}^{2}\right]$ & $27.0 \pm 4.4$ \\
\hline Women & $7(54 \%)$ \\
\hline $\mathrm{CHADS}_{2}$ score & $1.2 \pm 1.3$ \\
\hline $\mathrm{CHA}_{2} \mathrm{DS}_{2}$-VASc score & $1.92 \pm 1.7$ \\
\hline HAS-BLED score & $0.85 \pm 1.1$ \\
\hline \multicolumn{2}{|l|}{ Atrial rhythm (including history) } \\
\hline $\begin{array}{l}\text { Atrial fibrillation (paroxysmal } \\
\text { or permanent) }\end{array}$ & $0(0 \%)$ \\
\hline Atrial flutter & $0(0 \%)$ \\
\hline \multicolumn{2}{|l|}{ Clinical features } \\
\hline Prior ischemic stroke & $3(23 \%)$ \\
\hline Coronary artery disease & $0(0 \%)$ \\
\hline Valvular heart disease & $0(0 \%)$ \\
\hline $\begin{array}{l}\text { Renal function (mean eGFR, } \\
\text { in } \mathrm{mL} / \mathrm{min} / 1.73 \mathrm{~m}^{2} \text { ) }\end{array}$ & $97 \pm 24$ \\
\hline \multicolumn{2}{|l|}{ Anti-thrombotic therapy prior to laac } \\
\hline Acetylsalicylic acid & $3(23 \%)$ \\
\hline $\begin{array}{l}\text { Platelet inibitors other than } \\
\text { acetylsalicylic acid }\end{array}$ & $0(0 \%)$ \\
\hline Chronic oral anticoagulation & $0(0 \%)$ \\
\hline \multicolumn{2}{|l|}{ Echocardiographic data } \\
\hline $\begin{array}{l}\text { Systolic left ventricular } \\
\text { ejection fraction [\%] }\end{array}$ & $65 \pm 2$ \\
\hline Left atrial diameter (PLAX echo, mm) & $42 \pm 5$ \\
\hline
\end{tabular}

Values are number (\%) or mean \pm standard deviation; eGFR estimated glomerular filtration rate; PLAX — parasternal long axis

Table 2. Intervention $(n=13)$.

\begin{tabular}{lc}
\hline Procedural characteristics & \\
Flouroscopy time [min] & $16 \pm 8$ \\
Amplatzer Cardiac Plug & $6(20 \pm 3$, \\
& $16-24 \mathrm{~mm})$ \\
Amplatzer Amulet Occluder & $7(21 \pm 4$, \\
& $16-25 \mathrm{~mm})$ \\
Amplatzer Septal Occluder & $10(34 \pm 6$, \\
& $24-40 \mathrm{~mm})$ \\
Amplatzer Cribriform Occluder & $1(30 \mathrm{~mm})$ \\
Amplatzer PFO Occluder & $2(25 \mathrm{~mm})$ \\
Implantation attempts & $1.0 \pm 0.0$ \\
Concomittant procedures & $12(92 \%)$ \\
Coronary angiography & \\
Perirocedural success & \\
and adverse events & $13(100 \%)$ \\
Procedural success & $0(0 \%)$ \\
Combined safety endpoint* & \\
\hline
\end{tabular}

*Composite of death, stroke, clinically relevant pericardial effusion, VARC-2 life-threatening or major bleeding, VARC-2 major access vessel complication, device embolization, VARC-2 severe kidney injury, or bailout surgery. Values are number (\%) or mean \pm standard deviation; PFO - patent foramen ovale; VARC — Valve Academic

Research Consortium

\section{Statistical analysis}

Data are expressed as mean and standard deviation for continuous variables and as number and percentage for categorical variables. Analysis was performed using a standard statistical software program (SPSS, version 23; IBM, Armonk, NY, USA).

\section{Results}

\section{Baseline characteristics}

Thirteen patients were included between April 2013 and June 2015. Patients were $58 \pm 9$ years old (54\% women). Baseline characteristics are described in Table 1 . No patient had any history of AF. Three patients had a previous history of ischemic cerebrovascular events. The total procedural fluoroscopy time was $16 \pm 8$ min (Table 2).

\section{Device success}

In all patients ASD or PFO and LAA closures were successful. In 1 patient, 2 ASDs and in 1 patient, a PFO in addition to an ASD were present and closed with 2 separate devices (Figs. 2,3). No periprocedural complications occurred (Table 2).

\section{Clinical follow-up}

Length of hospital stay was 1 night in 9 patients and the remaining 4 patients were discharged the same day. TEE follow-up was available after $6.7 \pm 2.0$ months in 10 patients. No device-related complications occurred (Table 3). One patient needed a re-intervention because of 2 residual ASD shunts at follow up. Re-intervention was successfully performed under fluoroscopy only using a $25 \mathrm{~mm}$ and a $18 \mathrm{~mm}$ PFO Occluder at 6 months after initial procedure. At clinical follow-up of $2.0 \pm 0.8$ years, no adverse events had occurred, no patient had reached a primary safety or efficacy endpoint, and at latest contact $91 \%$ of patients were on no anticoagulant or antiplatelet medication and 9\% still acetylsalicylic acid (Table 4). The patients were asked for clinical symptoms of AF and all postinterventional ECGs were screened for AF. No patient had developed AF.

\section{Discussion}

Atrial fibrillation is the most common sustained cardiac arrhythmia [25] and is associated with substantial mortality and morbidity, particularly due to stroke [26]. ASD closure is advocated for prevention of stroke (paradoxical or secondary to AF) or heart failure due to volume overload. $\mathrm{PFO}$ closure is used for prevention of paradoxical 
Table 3. Postprocedural data and follow-up transesophageal echocardiography (TEE).

\begin{tabular}{lc}
\hline Post procedure in-hospital $(\mathbf{n}=\mathbf{1 3})$ & \\
Hospital stay [days] & $0.8 \pm 0.4$ \\
Same day discharge & $4(31 \%)$ \\
Pericardial effusion & $0(0 \%)$ \\
Antithrombotic therapy $(\mathbf{n}=\mathbf{1 3})$ & \\
Acetylsalicylic acid & $12(92 \%)$ \\
Platelet inibitors other than & $12(92 \%)$ \\
acetylsalicylic acid & $12(92 \%)$ \\
Dual antiplatelet therapy & $0(0 \%)$ \\
Vitamin K antagonists & $0(0 \%)$ \\
Non vitamin K dependant & \\
oral anticoagulants & \\
TEE (n = 10) & $6.7 \pm 2.0$ \\
Duration from intervention [months] & $10(100 \%)$ \\
All devices in correct position & $0(0 \%)$ \\
Late pericardial effusion & $0(0 \%)$ \\
Late device embolization & $0(0 \%)$ \\
Device thrombus (any) & $8(100 \%)$ \\
Procedural success at TEE follow-up & $1(10 \%)$ \\
Shunt or leak $>5$ mm & $2(20 \%)$ \\
Shunt or leak < 5 mm & \\
\hline
\end{tabular}

Values are number $(\%)$ or mean \pm standard deviation.

Table 4. Clinical follow-up $(n=13)$.

\begin{tabular}{lc}
\hline Long-term clinical follow-up & $13(100 \%)$ \\
Duration from procedure [years] & $2.0 \pm 0.8$ \\
Antithrombotic therapy & \\
Acetylsalicylic acid & $1(9 \%)$ \\
Other platelet inibitors & $0(0 \%)$ \\
Dual antiplatelet therapy & $0(0 \%)$ \\
Vitamin K antagonists & $0(0 \%)$ \\
Non vitamin K dependant & $0(0 \%)$ \\
oral anticoagulants & \\
Clinical outcome & \\
All-cause death & $0(0 \%)$ \\
All-cause stroke & $0(0 \%)$ \\
All-cause transient ischemic attack & $0(0 \%)$ \\
Myocardial infarction & $0(0 \%)$ \\
Systemic embolism & $0(0 \%)$ \\
Venous thrombosis/ & $0(0 \%)$ \\
/pulmonary embolism & \\
Fatal, lifethreatening, or major bleeding & $0(0 \%)$ \\
Hospitalization & $0(0 \%)$ \\
Primary safety endpoint & $0(0 \%)$ \\
Primary efficacy endpoint & $0(0 \%)$ \\
\hline
\end{tabular}

Values are number $(\%)$ or mean \pm standard deviation. stroke. LAA closure is indicated for prevention of stroke or bleeding in patients with AF [13-18].

The present study reports the feasibility and safety of "primary primary" preventive LAA closure during percutaneous ASD or $\mathrm{PFO}$ closure in patients with enlarged atria but no AF. It proved technically successful and safe but efficacy of our approach cannot be determined. No patient developed AF and therefore the potential of preventing embolic ischemia or bleeding because of the obviated need for oral anticoagulation remains unknown.

A large nationwide study of Nyboe et al. [21] included 1168 patients with ASD who were followed-up over a long-term period. The risk of developing $\mathrm{AF}$ was up to 10 -fold in comparison to the general population. In case of conservative management, the stroke risk was 2.6 -fold. In case of ASD closure, the stroke risk was reduced to 2 -fold. This can be due to both, the elimination of paradoxical embolism and the reduction of atrial sizes. Yet, the risk of AF-associated stroke remains elelvated [21].

Atrial fibrillation with the need for COA is expected to occur with a certain frequency in patients at some point in time after septal defect closure while they were still in sinus rhythm [9, 27]. Age $>40$ years is a well-known risk factor for AF after ASD closure [28]. Moreover, ASD closure devices can trigger new-onset AF in the early phase. In the later phase they may reduce the risk of $\mathrm{AF}$ as the atria shrink after elimination of the shunt $[10,11]$. Large devices in the interatrial septum may render percutaneous LAA closure intricate or downright impossible.

Chronic oral anticoagulation has been proven to effectively prevent thromboembolic strokes but there is a risk of serious bleeding which deters many patients from taking this therapy [29]. Therefore, alternative treatment options for stroke prevention in patients with $\mathrm{AF}$ with increased stroke risk are needed. Closure of the LAA has emerged as promising [13, 30].

Left atrial appendage closure in the sense of "primary primary" prevention has the potential to avoid future strokes in case new-onset $\mathrm{AF}$ goes undetected on top of the potential to avoid major bleeding in case a patient requires $\mathrm{COA}$ for $\mathrm{AF}$ during follow-up. However as is inherent to every preventive intervention, the risk and cost of the combined and partially preventive intervention may become unjustified, in case AF never develops during follow-up. This concern is alleviated by the safety of such an approach which is apparent from these data. 


\section{Limitations of the study}

The study has several limitations. It is a retrospective study and not a randomized trial and therefore selection bias is evident. The sample size is small. Furthermore, none of these patients developed AF during the relatively short observation period. More patients and longer follow-up are required to derive a clinically important message.

\section{Conclusions}

This study demonstrates feasibility of "primary primary" preventive LAA closure during percutaneous closure of large ASDs or PFOs with atrial enlargement in patients without AF. In this series no adverse safety events occurred. The idea is to prepare such patients for the likely event of later AF. ASD closure alone with a large septal device renders it difficult if not impossible to later perform a transseptal puncture for LAA closure.

Conflict of interest: S. Gloekler - grant from the Swiss Heart Foundation; F. Maisano - consultant for Abbott, Edwards, and Medtronic, and cofounder and shareholder of 4Tech Cardio and receiver of royalties from Edwards; B. Meier - speaker fees of Abbott; F. Nietispach - consultant for Abbott, Edwards, and Medtronic, and shareholder of Edwards.

\section{References}

1. Hoffman J, Kaplan S. The incidence of congenital heart disease. J Am Coll of Cardiol. 2002; 39(12): 1890-1900, doi: 10.1016/ s0735-1097(02)01886-7, indexed in Pubmed: 12084585.

2. Gatzoulis MA, Freeman MA, Siu SC, et al. Atrial arrhythmia after surgical closure of atrial septal defects in adults. N Engl J Med. 1999; 340: 839-846, doi: 10.1056/nejm199903183401103, indexed in Pubmed: 10080846.

3. Konstantinides S, Geibel A, Olschewski M, et al. A comparison of surgical and medical therapy for atrial septal defect in adults. N Engl J Med. 1995; 333(8): 469-473, doi: 10.1056/ nejm199508243330801, indexed in Pubmed: 7623878.

4. Gatzoulis M, Redington A, Somerville J, et al. Should atrial septal defects in adults be closed? Ann Thorac Surg. 1996; 61(2): 657-659, doi:10.1016/0003-4975(95)01043-2, indexed in Pubmed: 8572783.

5. Akula V, Durgaprasad R, Velam V, et al. Right ventricle before and after atrial septal defect device closure. Echocardiography. 2016; 33(9): 1381-1388, doi: 10.1111/echo.13250, indexed in Pubmed: 27109837.

6. Du ZD, Hijazi Z, Kleinman C, et al. Comparison between transcatheter and surgical closure of secundum atrial septal defect in children and adults: results of a multicenter nonrandomized trial. J Am Coll Cardiol. 2002; 39(11): 1836-1844, doi: 10.1016/s07351097(02)01862-4, indexed in Pubmed:12039500.

7. Fischer G, Stieh J, Uebing A, et al. Experience with transcatheter closure of secundum atrial septal defects using the Amplatzer septal occluder: a single centre study in 236 consecutive patients. Heart. 2003; 89(2): 199-204, doi: 10.1136/heart.89.2.199, indexed in Pubmed: 12527678.

8. Omeish A, Hijazi Z. Transcatheter closure of atrial septal defects in children \& adults using the Amplatzer Septal Occluder. J Interv Cardiol. 2001; 14(1): 37-44, doi: 10.1111/j.1540-8183.2001. tb00709.x, indexed in Pubmed: 12053325.

9. Spies C, Khandelwal A, Timmermanns I, et al. Incidence of atrial fibrillation following transcatheter closure of atrial septal defects in adults. Am J Cardiol. 2008; 102(7): 902-906, doi: 10.1016/j. amjcard.2008.05.045, indexed in Pubmed: 18805119.

10. Wagdi P. Incidence and predictors of atrial fibrillation following transcatheter closure of interatrial septal communications using contemporary devices. Clin Res Cardiol. 2010; 99(8): 507-510, doi: 10.1007/s00392-010-0149-3, indexed in Pubmed: 20352436.

11. Schneeberger Y, Schaefer A, Conradi L, et al. Minimally invasive endoscopic surgery versus catheter-based device occlusion for atrial septal defects in adults: reconsideration of the standard of care. Interact Cardiovasc Thorac Surg. 2016: ivw366, doi: 10.1093/icvts/ivw366, indexed in Pubmed:28040751.

12. Burow A, Schwerzmann M, Wallmann D, et al. Atrial fibrillation following device closure of patent foramen ovale. Cardiology. 2008; 111(1): 47-50, doi:10.1159/000113427, indexed in Pubmed: 18239392.

13. Holmes D, Reddy V, Turi Z, et al. Percutaneous closure of the left atrial appendage versus warfarin therapy for prevention of stroke in patients with atrial fibrillation: a randomised non-inferiority trial. Lancet. 2009; 374(9689): 534-542, doi: 10.1016/s01406736(09)61343-x, indexed in Pubmed: 19683639.

14. Tzikas A, Shakir S, Gafoor S, et al. Left atrial appendage occlusion for stroke prevention in atrial fibrillation: multicentre experience with the AMPLATZER Cardiac Plug. EuroIntervention. 2016; 11(10): 1170-1179, doi: 10.4244/eijy15m01_06, indexed in Pubmed: 25604089.

15. Nietlispach F, Gloekler S, Krause R, et al. Amplatzer left atrial appendage occlusion: Single center 10-year experience. Catheter Cardiovasc Interv. 2013; 82(2): 283-289, doi: 10.1002/ccd.24872, indexed in Pubmed: 23412815.

16. Ghenzi RA, Obeid S, Maisano F, et al. The evolving role of left atrial appendage occlusion: a high-volume single-centre experience. Cardiovasc Med. 2016; 19(11): 288-295, doi: 10.4414/ cvm.2016.00437.

17. Holmes D, Doshi S, Kar S, et al. Left atrial appendage closure as an alternative to warfarin for stroke prevention in atrial fibrillation. J Am Coll Cardiol. 2015; 65(24): 2614-2623, doi: 10.1016/j. jacc.2015.04.025, indexed in Pubmed: 26088300.

18. Reddy V, Sievert H, Halperin J, et al. Percutaneous left atrial appendage closure vs warfarin for atrial fibrillation. JAMA. 2014; 312(19): 1988-1998, doi:10.1001/jama.2014.15192, indexed in Pubmed: 25399274.

19. Wagdi P, Alkadhi H. Can computer tomography help predict feasibility of transseptal puncture after percutaneous closure of an interatrial septal communication? J Interv Card Electrophysiol. 2012; 34(2): 167-172, doi: 10.1007/s10840-011-9625-6, indexed in Pubmed: 22314668.

20. Zaker-Shahrak R, Meier B. Transseptal puncture for catheter ablation of atrial fibrillation after device closure of patent foramen ovale. Catheter Cardiovasc Interv. 2008; 71(4): 551-552, doi: 10.1002/ccd.21363, indexed in Pubmed: 18307237.

21. Nyboe C, Olsen MS, Nielsen-Kudsk JE, et al. Atrial fibrillation and stroke in adult patients with atrial septal defect and the long- 
term effect of closure. Heart. 2015; 101(9): 706-711, doi: 10.1136/ heartjnl-2014-306552, indexed in Pubmed: 25691512.

22. Praz F, Wahl A, Schmutz M, et al. Safety, feasibility, and longterm results of percutaneous closure of atrial septal defects using the Amplatzer septal occluder without periprocedural echocardiography. J Invasive Cardiol. 2015; 27(3): 157-162, indexed in Pubmed: 25740969.

23. Meier B, Blaauw Y, Khattab AA, et al. EHRA/EAPCI expert consensus statement on catheter-based left atrial appendage occlusion. Europace. 2014; 16(10): 1397-1416, doi: 10.1093/europace/ euu174, indexed in Pubmed: 25169595.

24. Kappetein AP, Head SJ, Généreux P, et al. Updated standardized endpoint definitions for transcatheter aortic valve implantation: the Valve Academic Research Consortium-2 consensus document. J Am Coll Cardiol. 2012; 60: 1438-1454, doi: 10.4244/EIJV8I7A121, indexed in Pubmed: 23022744.

25. Go A, Hylek E, Phillips K, et al. Prevalence of diagnosed atrial fibrillation in adults: national implications for rhythm management and stroke prevention: the AnTicoagulation and Risk Factors in Atrial Fibrillation (ATRIA) Study. JAMA. 2001; 285(18): 2370-2375, doi: 10.1001/jama.285.18.2370, indexed in Pubmed: 11343485.
26. De Backer O, Arnous S, Ihlemann N, et al. Percutaneous left atrial appendage occlusion for stroke prevention in atrial fibrillation: an update. Open Heart. 2014; 1(1): e000020, doi: 10.1136/ openhrt-2013-000020, indexed in Pubmed: 25332785.

27. Rigatelli G, Pedon L, Zecchel R, et al. Long-term outcomes and complications of intracardiac echocardiography-assisted patent foramen ovale closure in 1,000 consecutive patients. J Interv Cardiol. 2016; 29(5): 530-538, doi: 10.1111/joic.12325, indexed in Pubmed: 27500752.

28. Giardini A, Donti A, Sciarra F, et al. Long-term incidence of atrial fibrillation and flutter after transcatheter atrial septal defect closure in adults. Int J Cardiol. 2009; 134(1): 47-51, doi: 10.1016/j. ijcard.2008.02.003, indexed in Pubmed: 18396341.

29. Landmesser U, Holmes DR. Left atrial appendage closure: a percutaneous transcatheter approach for stroke prevention in atrial fibrillation. Eur Heart J. 2012; 33(6): 698-704, indexed in Pubmed: 22041550.

30. Fountain R, Holmes D, Chandrasekaran K, et al. The PROTECT AF (WATCHMAN Left atrial appendage system for embolic PROTECTion in patients with atrial fibrillation) trial. Am Heart J. 2006; 151(5): 956-961, doi: 10.1016/j.ahj.2006.02.005, indexed in Pubmed: 16644311. 